\title{
On the friction coefficient of straight-chain aggregates
}

\author{
Lorenzo Isella ${ }^{\mathrm{a}, 1}$, Yannis Drossinos ${ }^{\mathrm{a}, \mathrm{b}, *}$ \\ ${ }^{a}$ European Commission, Joint Research Centre, I-21027 Ispra (VA), Italy \\ ${ }^{b}$ School of Mechanical \& Systems Engineering, Newcastle University, Newcastle upon \\ Tyne NE1 7RU, United Kingdom
}

\begin{abstract}
A methodology to calculate the friction coefficient of an aggregate in the continuum regime is proposed. The friction coefficient and the monomer shielding factors, aggregate-average or individual, are related to the moleculeaggregate collision rate that is obtained from the molecular diffusion equation with an absorbing boundary condition on the aggregate surface. Calculated friction coefficients of straight chains are in very good agreement with previous results, suggesting that the friction coefficients may be accurately calculated from the product of the collision rate and an average momentum transfer, the latter being independent of aggregate morphology. Langevin-dynamics simulations show that the diffusive motion of straightchain aggregates may be described either by a monomer-dependent or an aggregate-average random force, if the shielding factors are appropriately chosen.
\end{abstract}

Keywords: Straight-chain aggregates, friction coefficient, diffusion coefficient, collision rate, Langevin simulations

\section{Introduction}

The hydrodynamic drag on a fractal aggregate suspended in a viscous fluid determines many of its dynamical properties, such as sedimentation, agglomeration, and mobility (diffusive, mechanical, electrical) [1]. Fractal

\footnotetext{
* Corresponding author.

Email addresses: lorenzo.isella@isi.it (Lorenzo Isella), ioannis.drossinos@jrc.ec.europa.eu (Yannis Drossinos)

${ }^{1}$ Present address: ISI Foundation, Turin 10133, Italy.
} 
aggregates arise from the agglomeration of smaller, primary spherules, hereafter called monomers, that do not coalesce, but rather retain their identity in the resulting aggregate. The calculation of the drag force can be a formidable task, often requiring simplifying assumptions like spherical [2, 3] or ellipsoidal symmetry [4].

The friction coefficient of non-spherical aggregates in the continuum regime (fluid mean free path much smaller than the monomer radius $R_{1}$ ) has been calculated by treating the fractal aggregate as a porous particle in a viscous creeping flow (Stokes flow) of constant [4] or variable [2, 3, 5] permeability. Alternatively, Filippov [6] performed a multipole expansion of the Stokes flow velocity with slip and non-slip boundary conditions on the aggregate surface. Happel and Brenner [7] calculated the hydrodynamic forces on a collection of spheres by the method of reflections obtaining a slowly converging series. In these calculations the hydrodynamic force was obtained by integrating the fluid stress tensor over the aggregate surface. For straight chains, Dahneke [8], based on an extrapolation of experimental data, proposed an empirical correlation for the friction forces parallel and perpendicular to the chain symmetry axis.

Herein, we propose a versatile, albeit approximate, procedure to determine the friction coefficient of an aggregate in creeping flows in the continuum regime. We argue that the friction coefficient of a fractal-like aggregate may be related to the ratio of two molecular collision rates: the molecule-aggregate collision rate and the molecule-monomer collision rate. These molecular collision rates are calculated from the diffusion equation for the fluid density with appropriate boundary conditions. Hence, we suggest that the friction coefficient of fractal-like objects may be accurately (but approximately) calculated from the solution of the Laplace equation (with appropriate boundary conditions) for the fluid density, without requiring the solution of the Stokes equations for the fluid velocity.

The relationship between collision rates and friction coefficient allows us to introduce the monomer shielding factor (either individual or aggregateaverage) that provides a measure of monomer shielding within an aggregate. We apply the proposed methodology to calculate the friction coefficients of straight-chain aggregates. The calculated friction coefficients are compared to previous analytical results and numerical results to justify the approximations made a posteriori, and to validate the proposed methodology. We remark, however, that the methodology is general enough to be applicable to the calculation of friction coefficient of a general fractal-like aggregate. 
Calculated shielding factors of individual monomers in straight chains composed of $k=5,8$ monomers are used to obtain the chain diffusion coefficient by Langevin-dynamics simulations. These coefficients are found to be in very good agreement with the diffusion coefficients obtained from the ratio of molecular collision rates.

Such Langevin simulations of aggregate formation and motion, which depend explicitly on the inter-monomer interaction potential, offer a potentially useful tool to investigate the effect of inter-monomer forces on agglomeration dynamics and the shape of the resulting aggregate. Previous works have suggested, either experimentally or numerically, the importance of inter-particle forces. For example, Chakrabarty el al. [9] hypothesized that electic forces were responsible for the unexpectedly low fractal dimension (between 1.2 and 1.5) of a minority of soot aggregates formed in a premixed ethene-oxygen flame. di Stasio et al. [10], in their experimental and numerical studies of the kinetics of agglomeration and growth of soot nanoparticles in an ethylene-air diffusion flame, suggested that the number of collisions is enhanced due to the attractive van der Waals forces. Kostoglou and co-workers [11, 12] studied theoretically the time-dependent evolution of aggregate morphology, as described by the aggregate fractal dimension. Their analyses was based on a proposed constitutive law for the agglomeration of two aggregates, a law that depends on the inter-monomer (and hence inter-aggregate) interaction potential. The Langevin simulations described herein and in, e.g., Ref. [13], could address these theoretical and experimental observations by providing a technique to validate them.

\section{Molecule-aggregate collision rate and friction coefficient}

\subsection{Methodology}

The motivation of our work stems from experimental measurements of the mass transfer (attachment) coefficient of gas molecules to nanoparticles of a variety of species, shapes, and sizes [14, 15]. The rate of transfer of gas molecules of density $\rho$ to identical aggregates composed of $k$ monomers (k-aggregates) of concentration $N_{k}$ is

$$
\frac{d \rho}{d t}=-\tilde{K}_{k} N_{k} \rho
$$

where $\tilde{K}_{k}$ is the total mass transfer coefficient of fluid molecules per aggregate, namely $\tilde{K}_{k}=h_{k} S_{k}$ where $h_{k}$ is the average heat transfer coefficient 
as usually defined in the chemical engineering literature (per unit surface area) and $S_{k}$ the geometric surface area of the aggregate. Equation (1) is valid for a molecule-aggregate sticking coefficient of unity. Such mass transfer measurements may be performed by labelling atoms or molecules, via, for example, radioactive labelling (epiphaniometer) or electrical charging (diffusion charger), and subsequently detecting the atoms or molecules attached to the aggregate. Siegmann and Siegmann [14, who measured total mass transfer coefficients over a wide range of aggregate mobilities via diffusive electrical charging, argued that the product of the mass transfer coefficient times the aggregate (electrical) mobility $B_{k}$ is approximately constant

$$
\tilde{K}_{k} \times B_{k}=\text { constant },
$$

where $B_{k}$, the aggregate mobility, is the ratio of aggregate terminal velocity to the steady-state force causing it [16].

Keller et al. 15] presented further experimental measurements supporting the claim that Eq. (2) hold (within experimental error) for a variety of experimental conditions and aggregate shapes. They suggested that Eq. (2) constitutes an "empirical scaling law". They justified it by arguing that both the mass transfer coefficient and the aggregate mobility depend on the aggregate surface area exposed to the fluid. They referred to the particle surface area upon which mass, momentum, and energy transfer are dominant as their active surface area. Accordingly, the mass transfer coefficient is proportional to the particle active surface area, whereas the mobility is inversely proportional rendering their product a constant [14, 15]. In particular, the total mass transfer coefficient $\tilde{K}_{k}$ to a sphere in the continuum regime is proportional to its diameter $d_{p}$, whereas in the free molecular regime it is proportional to its geometric surface area $\left(\sim d_{p}^{2}\right)$.

If multiple scattering events are neglected (sticking coefficient of unity), the attachment rate $\tilde{K}_{k} N_{k}$ is proportional to the molecule-aggregate collision rate. For Stokes drag the mobility of a $k$-aggregate is inversely proportional [16] to the aggregate friction coefficient $f_{k}$. Equation (2), then, implies that the collision rate $K_{k}$ between a molecule and a $k$-aggregate is proportional to the friction coefficient, $K_{k}=c f_{k}$. The proportionality constant may be evaluated by considering, as experimental measurements suggest, that Eq. (2) is approximately valid for $k=1$. At steady state and for a spherical monomer, the integral molecule-monomer collision rate $K_{1}$ is calculated from the the gas diffusion equation with an absorbing boundary 
condition on the monomer surface. Specifically,

$$
K_{1}=\int_{S_{1}} \mathbf{J}_{1} \cdot d \mathbf{S}=\int_{S_{1}} \mathbf{J}_{1} \cdot \hat{\mathbf{s}} d S=4 \pi D_{g} R_{1} \rho_{\infty} \quad,
$$

where the diffusive flux is $\mathbf{J}_{1}=-D_{g} \nabla \rho$ with $\rho(r)=\rho_{\infty}\left(1-R_{1} / r\right), \rho_{\infty}$ is the gas-phase (fluid) density far away from the aggregate, and $D_{g}$ the gas selfdiffusion coefficient (see, for example, Ref. [1]). The monomer surface area is denoted by $S_{1}, \hat{\mathbf{s}}$ is the unit vector orthogonal to the monomer surface, and $d S$ the surface element $\left(d S=R_{1}^{2} \sin \theta d \theta d \phi\right)$.

The Stokes friction coefficient of an isolated monomer is $f_{1}=m_{1} \beta_{1}=$ $6 \pi \mu_{g} R_{1}$ with $\mu_{g}$ the fluid viscosity, leading to the proportionality constant $c=2 D_{g} \rho_{\infty} /\left(3 \mu_{g}\right)$ (for a similar calculation of the particle active surface area in the continuum regime cf. Ref. [17]). It is more convenient to eliminate the proportionality constant by considering the drag force $f_{k}$ relative to the total drag on $k$ isolated monomers $f_{1}$,

$$
\frac{K_{k}}{k K_{1}}=\frac{f_{k}}{k f_{1}}
$$

Theoretical arguments provide partial support of Eqs. (2), (4). In kinetic theory the mean force exerted on a particle by fluid molecules is the product of the molecule-particle collision rate times the mean momentum transfer per collision [18. The main approximation of our work, and the approximation implicit in Eqs. (2), (4), is that the dominant contribution to the friction force arises from the collision rate. If the mean momentum transfer is taken to be independent of aggregate morphology the ratio of the friction coefficients $f_{k} /\left(k f_{1}\right)$ becomes the appropriate ratio of collision rates shown in Eq. (4). It is, thus, apparent that Eq. (4) is approximate. The friction force arises from the momentum transfer during molecule-particle collisions; even if the sticking probability is unity not all collisions transfer the same momentum since gas velocities are distributed according to a probability density function that may, in principle, be calculated from the Boltzmann equation.

The Stokes friction coefficient of a $k$-aggregate is expressed as $f_{k}=k m_{1} \beta_{k}$ where $\beta_{k}$ is the average friction coefficient per unit monomer mass and $k m_{1}$ the aggregate mass [13. Equation (4) then leads to our main result that relates the friction coefficient of a general-shaped aggregate to the ratio of two molecular collision rates

$$
\frac{K_{k}}{k K_{1}}=\frac{\beta_{k}}{\beta_{1}} \equiv \eta_{k} .
$$


The last equality defines the average monomer shielding factor $\eta_{k}$ as the ratio of the average friction coefficient of a monomer in an aggregate to the friction coefficient of an isolated monomer. The shielding factor provides a measure of the shielding of a monomer by other monomers in an aggregate; as such, it depends on aggregate morphology. It has been used to calculate corrections of the Stokes drag on a porous medium consisting of identical non-interacting spheres [19], in modifications of heat transfer to an aggregate due to monomer shielding [20], and it has been related to the aggregate diffusion coefficient [13]. The friction coefficient, calculated according to Eq. (5), naturally leads to the Stokes-Einstein diffusion coefficient $D_{k}$ of a $k$-aggregate via

$$
D_{k}=\frac{k_{B} T}{k m_{1} \beta_{k}}=D_{1} \frac{1}{k \eta_{k}}
$$

where the Stokes-Einstein monomer diffusion coefficient is $D_{1}\left[=k_{B} T /\left(m_{1} \beta_{1}\right)\right]$. Furthermore, the aggregate mobility radius, defined as $D_{k} \equiv k_{B} T /\left(6 \pi \mu_{g} R_{k}\right)$, is

$$
\frac{R_{k}}{R_{1}}=k \eta_{k}
$$

For the ideal aggregates defined in Ref. [13], aggregates in the free-draining approximation whereby the hydrodynamic forces on a monomer are independent of its state of aggregation, $\eta_{k}=1$. The shielding factor also gives the ratio of a $k$-aggregate active surface area to the active surface area of $k$ isolated monomers.

The dynamic shape factor of particles of arbitrary shape, a correction factor used to account for the effect of shape on particle motion, is also related to the average monomer shielding factor. The aggregate dynamic shape factor $\chi_{s}$ is defined by

$$
\chi_{k}=\frac{f_{k}}{6 \pi \mu_{g} R_{e q}},
$$

where the $R_{e q}$ is the radius of the equivalent volume sphere, $R_{e q}=k^{1 / 3} R_{1}$. Hence,

$$
\chi_{s}=\eta_{k} k^{2 / 3} .
$$

As in the case of an isolated monomer, the integral collision rate may be calculated from the fluid diffusion equation in steady state $\left[\nabla^{2} \rho(\mathbf{r})=0\right]$, with an absorbing boundary condition on the aggregate surface $\left[\rho\left(\mathbf{r}_{\text {sur }}\right)=0\right.$, neglect of multiple scattering events] and constant density far away from the 
aggregate. $\left(\rho \rightarrow \rho_{\infty}\right.$ for $\left.|\mathbf{r}| \rightarrow \infty\right)$. The diffusive flux $\mathbf{J}_{k}=-D_{g} \nabla \rho$ yields the collision rate for a generic $k$-aggregate

$$
K_{k}=\int_{S} \mathbf{J}_{k} \cdot d \mathbf{S}=\int_{S} \mathbf{J}_{k} \cdot \hat{\mathbf{s}} d S,
$$

where $S$ is the aggregate surface, $\hat{\mathbf{s}}$ the unit vector orthogonal to the aggregate surface, and $d S$ the surface element [see, also, Eq. (3)]. This calculation of the collision rate reflects the experimental procedure to measure the attachment coefficient: the total mass transfer rate is proportional to the integral collision rate. Equation (10) highlights our approximations in that the friction coefficient is approximately related to a surface integral of the molecular diffusive flux instead of the stress tensor. It is worthwhile noting that the linearized Bhatnagar-Gross-Krook equation (BGK), a single relaxation-time approximation of the Boltzmann equation, leads to a selfdiffusion coefficient inversely proportional to the BGK velocity-independent collision frequency [21].

The proposed methodology suggests that the friction coefficient of fractallike aggregates may be accurately calculated from the solution of the scalar Laplace equation for the fluid density (with appropriate boundary conditions) without solving the Stokes equations. Once the fluid density has been determined the collision rates may be easily calculated during post-processing via Eq. (10). On the other hand, the Stokes equations (linear equations as the Laplace equation) must be solved for the three components of the fluid velocity to calculate the drag force. This replacement, in addition to its theoretical implications, has considerable computational advantages that depend primarily on the symmetry of the aggregate and the dimensionality of the system. Specifically, if axisymmetric aggregates are considered, as in this work, the Stokes equations must be solved for the two components of the drag force. The Laplace equation, however, must be solved only once for the fluid density in two dimensions.

\subsection{Friction coefficient of straight-chain aggregates}

Equations (5) and (10) will be used to calculate average and individual monomer shielding factors and the friction coefficient of straight monomer chains via the steady-state collision rate $K_{k}$ to a single aggregate. We stress that Eq. (5) is general enough, and easy to implement numerically, to be applicable to a generic fractal-like aggregate. A straight-chain aggregate is cylindrically symmetric, suggesting that a friction coefficient along the axis 
orthogonal to the axis of symmetry $\beta_{k}^{\perp}$ and parallel to it $\beta_{k}^{\|}$may be defined as

$$
\frac{K_{k}^{\perp}}{k K_{1}^{\perp}}=\frac{\beta_{k}^{\perp}}{\beta_{1}} \equiv \eta_{k}^{\perp} \quad, \quad \frac{K_{k}^{\|}}{k K_{1}^{\|}}=\frac{\beta_{k}^{\|}}{\beta_{1}} \equiv \eta_{k}^{\|} .
$$

The anisotropic collision rates $K_{k}^{\perp(\|)}$ are obtained by projecting the diffusive flux $\mathbf{J}_{k}$ parallel and perpendicular to the symmetry axis, and integrating its absolute value over the aggregate surface, cf. Eq. (10),

$$
\begin{gathered}
K_{k}^{\|} \equiv \int_{S}\left|J_{k}^{\|}\right| d S=\int_{S}\left|\mathbf{J}_{k} \cdot \hat{\mathbf{s}}_{\|}\right| d S=\int_{S}\left|D_{g} \frac{\partial \rho}{\partial z}\right| d S \\
K_{k}^{\perp} \equiv \int_{S}\left|J_{k}^{\perp}\right| d S=\int_{S}\left[\left|\mathbf{J}_{k} \cdot \hat{\mathbf{s}}_{\perp, 1}\right|^{2}+\left|\mathbf{J}_{k} \cdot \hat{\mathbf{s}}_{\perp, 2}\right|^{2}\right]^{1 / 2} d S \\
=\int_{S}\left[\left|\mathbf{J}_{k}^{x}\right|^{2}+\left|\mathbf{J}_{k}^{y}\right|^{2}\right]^{1 / 2} d S=\int_{S} d S\left[\left(D_{g} \frac{\partial \rho}{\partial x}\right)^{2}+\left(D_{g} \frac{\partial \rho}{\partial y}\right)^{2}\right]^{1 / 2}
\end{gathered}
$$

where (in three dimensions and in a Cartesian co-ordinate system) the unit vectors are $\hat{\mathbf{s}}_{\|}=\hat{\mathbf{s}}_{z}, \hat{\mathbf{s}}_{\perp, 1}=\hat{\mathbf{s}}_{x}$, and $\hat{\mathbf{s}}_{\perp, 1}=\hat{\mathbf{s}}_{y}$ with the $z$ coordinate along the symmetry axis and $x, y$ the co-ordinates perpendicular to it. The absolute value is necessary in Eq. (12) to ensure that the collision rate is non-zero. As expected, Eq. (11) shows that an isolated spherical monomer has only one isotropic friction coefficient, $\beta_{k}^{\perp}=\beta_{k}^{\|}=\beta_{1}$.

According to Eq. (11) the parallel and perpendicular fluxes to a spherical monomer should be calculated. Even though a sphere is isotropic once a symmetry axis is randomly chosen the two fluxes differ, $K_{1}^{\perp} \neq K_{1}^{\|}$. Let the symmetry axis be the $z$-axis. Since its choice is arbitrary (due to spherical symmetry)

$$
\int_{S}\left|J_{1}^{x}\right| d S=\int_{S}\left|J_{1}^{y}\right| d S=\int_{S}\left|J_{1}^{z}\right| d S=2 \pi D_{g} R_{1} \rho_{\infty} .
$$

However, the perpendicular flux depends on the two fluxes in the two perpendicular directions. Specifically, the (local) molecular fluxes at the monomer surface are $J_{1}^{\|}=J_{1}^{z}=J_{1} \cos \theta$ and $J_{1}^{\perp}=\left(\left|\mathbf{J}_{1}^{x}\right|^{2}+\left|\mathbf{J}_{1}^{y}\right|^{2}\right)^{1 / 2}=J_{1}|\sin \theta|$ with $\left.J_{1}\right|_{r=R_{1}}=D_{g} \rho_{\infty} / R_{1}$, cf. Eq. (3). Then, the analytical evaluation of the diffusional fluxes on the surface of the monomer gives

$$
K_{1}^{\|}=\int_{S}\left|J_{1}^{\|}\right| d S=D_{g} \rho_{\infty} R_{1} \int_{0}^{2 \pi} d \phi \int_{0}^{\pi} d \theta|\cos \theta| \sin \theta=2 \pi D_{g} R_{1} \rho_{\infty}
$$




$$
K_{1}^{\perp}=\int_{S}\left|J_{1}^{\perp}\right| d S=D_{g} \rho_{\infty} R_{1} \int_{0}^{2 \pi} d \phi \int_{0}^{\pi} d \theta \sin ^{2} \theta=\pi^{2} D_{g} R_{1} \rho_{\infty}
$$

Note that, as expected from Eq. $12 \mathrm{~b}, K_{1}^{\perp} \neq K_{1}^{\|}$(in fact, $K_{1}^{\perp}>K_{1}^{\|}$), and that $K_{1}^{\|}$is independent of the choice of the symmetry axis, as shown in Eq. (13). We stress that even though the parallel and perpendicular diffusional fluxes as defined differ for a spherical monomer, its diffusion coefficient is unique and it does not depend on the choice of the symmetry axis.

We calculated the friction coefficients (average, and for motion parallel and perpendicular to the symmetry axis) of straight chains consisting of up to $k=64$ monomers. The finite-element software Comsol Multiphysics [23] was used to solve the diffusion equation in cylindrical coordinates $(r, z)$ with an absorbing boundary condition on the chain surface. In this co-ordinate system $J_{k}^{\|}=J_{k}^{z}$ and $J_{k}^{\perp}=J_{k}^{r}$. We tested the mesh-independence of the solutions. The size of the cylindrical computational domain was at least two orders of magnitude larger than the corresponding dimension of the chain to ensure that the condition $\rho_{\infty}=$ const hold at the computational-domain boundaries. The calculated diffusive flux to a single $k$-chain, and its components parallel and perpendicular to the symmetry axis, was numerically integrated over the aggregate surface to determine the molecule-aggregate collision rate.

Table 1 compares the friction coefficients obtained via the ratio of collision rates to previous analytical and numerical results. The agreement is very good, justifying a posteriori our main approximations. For example, explicit calculation of the difference between our results and those reported by Dahneke [8] for chains composed of $k=2,3,4,5,8$ monomers shows that the maximum difference is $0.88 \%, 3.40 \%$, and $1.38 \%$ in $\eta_{k}, \eta_{k}^{\|}$, and $\eta_{k}^{\perp}$, respectively.

Filippov [6] calculated the friction coefficient of non-overlapping spheres via a multipole expansion of the Stokes flow velocity in a series of spherical harmonics. The friction coefficient of a straight chain consisting of $k=8$ monomers was reported to be $\beta_{8}^{\perp} / \beta_{1}=0.435$ (in our notation), in good agreement with the calculated 0.428 , Table 1 . The other values in the second column of Table 1 were obtained from reported dynamic shape factors by inverting Eq. (9).

Dahneke [8], based on an interpolation of experimental measurements on chains with $k \leq 5$, provides extrapolation formulae for the dimensionless drag forces felt by a straight chain for motion parallel and perpendicular to the 


\begin{tabular}{ccccc}
\hline \hline $\begin{array}{c}\text { Friction } \\
\text { coefficient }\end{array}$ & $\begin{array}{c}\text { Filippov } \\
\text { Ref. [6] }\end{array}$ & $\begin{array}{c}\text { Happel Brenner } \\
\text { Ref. [7] }\end{array}$ & $\begin{array}{c}\text { Dahneke } \\
\text { Ref. [8] }\end{array}$ & $\begin{array}{c}\text { Collision } \\
\text { rate }\end{array}$ \\
\hline$\eta_{2}=\beta_{2} / \beta_{1}$ & & & 0.692 & 0.694 \\
$\eta_{3}=\beta_{3} / \beta_{1}$ & & & 0.569 & 0.574 \\
$\eta_{4}=\beta_{4} / \beta_{1}$ & & & 0.507 & 0.507 \\
$\eta_{5}=\beta_{5} / \beta_{1}$ & & & 0.461 & 0.463 \\
$\eta_{8}=\beta_{8} / \beta_{1}$ & & & 0.390 & 0.389 \\
& & & & \\
$\eta_{2}^{\|}=\beta_{2}^{\|} / \beta_{1}$ & & 0.645 & 0.639 & 0.633 \\
$\eta_{3}^{\|}=\beta_{3}^{\|} / \beta_{1}$ & & & 0.511 & 0.500 \\
$\eta_{4}^{\|}=\beta_{4}^{\|} / \beta_{1}$ & & & 0.442 & 0.430 \\
$\eta_{5}^{\|}=\beta_{5}^{\|} / \beta_{1}$ & & & 0.397 & 0.385 \\
$\eta_{8}^{\|}=\beta_{8}^{\|} / \beta_{1}$ & & & 0.324 & 0.313 \\
$\eta_{2}^{\perp}=\beta_{2}^{\perp} / \beta_{1}$ & 0.726 & 0.716 & 0.719 & 0.725 \\
$\eta_{3}^{\perp}=\beta_{3}^{\perp} / \beta_{1}$ & 0.613 & & 0.608 & 0.612 \\
$\eta_{4}^{\perp}=\beta_{4}^{\perp} / \beta_{1}$ & 0.550 & & 0.545 & 0.547 \\
$\eta_{5}^{\perp}=\beta_{5}^{\perp} / \beta_{1}$ & 0.508 & & 0.504 & 0.503 \\
$\eta_{8}^{\perp}=\beta_{8}^{\perp} / \beta_{1}$ & 0.435 & & 0.434 & 0.428 \\
\hline \hline
\end{tabular}

Table 1: Comparison of calculated friction coefficients of straight chains of $k=2-8$ monomers (average, and for motion parallel and perpendicular to the chain symmetry axis) with previous analytical and numerical results. The values for $\eta_{2}^{\perp}-\eta_{5}^{\perp}$ in the second column where obtained from reported values of the dynamic shape factor via Eq. (9).

symmetry axis. A straight chain composed of $k$ monomers was approximated as an ellipsoid of aspect ratio $k$. In our notation the interpolation formulae read

$$
\begin{aligned}
\eta_{k}^{\|(\perp)} & =\frac{\beta_{k}^{\|(\perp)}}{\beta_{1}} \\
& =\frac{A_{\|(\perp)}\left(k^{2}-1\right)}{6 \pi k} \cdot\left[\frac{2\left(k^{2}-1\right) \pm 1}{\sqrt{k^{2}-1}} \ln \left(k+\sqrt{k^{2}-1}\right)+B_{\|(\perp)} k\right]^{-1}
\end{aligned}
$$

where the positive (negative) sign is chosen for the parallel (perpendicular) coefficient. The formulae presented in Ref. 8] map to ours via $\eta_{k}^{\| \perp}=$ $c_{\|, \perp}(0) /(6 \pi k)$. We fitted our calculated anisotropic friction coefficients to 
Eqs. (15). The fits were performed with the Levenberg-Marquardt algorithm as implemented in the statistical language R Minpack library [22]. We found $A_{\perp}=51.07, B_{\perp}=0.8637, A_{\|}=27.45$, and $B_{\|}=-0.9386$ in excellent agreement with the fit reported by Dahneke [8] (see, also, Fig. 1).

Vainshtein et al. [4] performed an extensive analytical study of the equivalent mobility radii of oblate and prolate spheroids. Their results for nonporous impermeable prolate spheroids with aspect ratio $k$ expressed in our notation are

$$
\begin{aligned}
& \eta_{k}^{\perp}=\frac{\beta_{k}^{\perp}}{\beta_{1}}=\frac{8}{3 k}\left[\frac{k}{k^{2}-1}+\frac{2 k^{2}-3}{\left(k^{2}-1\right)^{3 / 2}} \ln \left(k+\sqrt{k^{2}-1}\right)\right]^{-1}, \\
& \eta_{k}^{\|}=\frac{\beta_{k}^{\|}}{\beta_{1}}=\frac{8}{3 k}\left[-\frac{2 k}{k^{2}-1}+\frac{2 k^{2}-1}{\left(k^{2}-1\right)^{3 / 2}} \ln \left(\frac{k+\sqrt{k^{2}-1}}{k-\sqrt{k^{2}-1}}\right)\right]^{-1} .
\end{aligned}
$$

Equations (16), expressed in terms of the mobility radius Eq. (7), are identical to the equations for the radius of the "equivalent radius" derived in Happel \& Brenner [7].

In the limit of large straight $k$-chains Eqs. 15,16 show that their perunit-mass friction coefficient tends to zero as $\beta_{k} \rightarrow 1 / \ln (2 k)$, whereas the chain friction coefficient becomes infinitely large as $k \beta_{k} \rightarrow k / \ln (2 k)$. The proportionality constant depends on the equations considered and on the choice of the constants in Eq. (15). Moreover, Eqs. (16) show that the parallel and perpendicular friction coefficients tend to $\beta_{k}^{\perp} \rightarrow 2 \beta_{k}^{\|}$as $k \rightarrow \infty$, whereas Eqs. 15 predict $\beta_{k}^{\perp} \rightarrow \beta_{k}^{\|} A_{\perp} / A_{\|}$with $A_{\perp} / A_{\|}=1.86$ [see, also, Eq. (19)].

In Fig. 1 we plot our numerical results (referred to as "Collision rate", circles), the numerical fit based on Eqs. (15) ("Collision rate fit", solid line), the anisotropic friction coefficients for an impermeable prolate spheroid according to Vainstein et al. 4], Eqs. (16), ("Vainstein et al.", dashed line), and the extrapolated results of Dahneke [8] ("Dahneke", dot-dashed line). Friction coefficients for motion parallel (left subfigure) and perpendicular to the chain symmetry axis are presented. In the right subfigure, squares denote results reported by Filippov [6] $(k=8)$ or derived from reported dynamic shape factors. As previously remarked the agreement of our calculated friction coefficients to those predicted by Eqs. (15) and 16 is very good.

Happel \& Brenner [7] presented a comprehensive analysis of the resistance coefficients of two equal-size spheres moving along the line of their centers or perpendicular to it. They solved the Stokes equations via the method of 

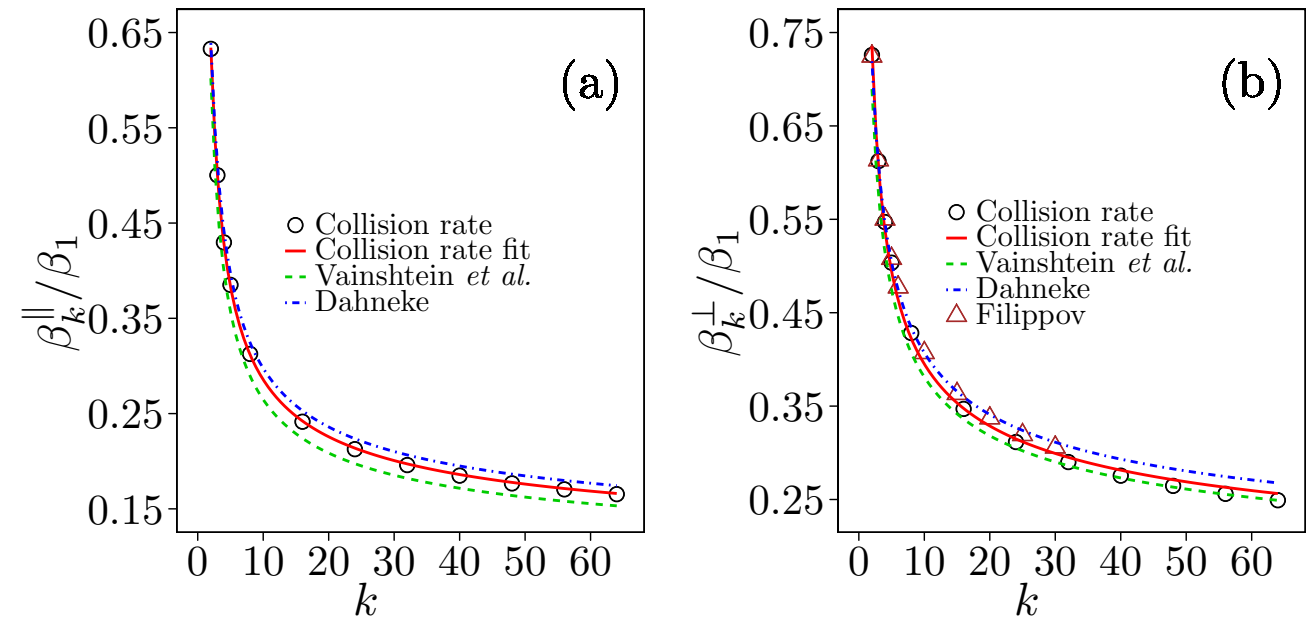

Figure 1: Calculated friction coefficients of straight chains $(k=2-64)$ for motion parallel (a) and perpendicular (b) to the chain symmetry axis plotted against the number of monomers. Comparison with previous results.

reflection. Their results for the friction coefficients of two equal-size nonrotating spheres as a function of their separation are compared to ours in Fig. 2. In particular, for two touching spheres their results $\beta_{2}^{\|} / \beta_{1}=0.645$ and $\beta_{2}^{\perp} / \beta_{1}=0.716$ compare favourably to ours, 0.633 and 0.725 , respectively. We remark that the comparison is best for touching spheres.

Table 2 presents calculated dynamic shape factors for the aggregates considered in our simulations. The shape factors where calculated via Eq. (9) by substituting the appropriate shielding factor $\left(\eta_{k}, \eta_{k}^{\|}, \eta_{k}^{\perp}\right)$. The last column presents the results of Ref. [6] determined from a multipole expansion of the Stokes velocity field.

Dahneke [8] also argued that for a large ensemble of identical straight chains undergoing random Brownian rotations the orientation-averaged friction coefficient would be

$$
\beta_{k}=\frac{3 \beta_{k}^{\|} \beta_{k}^{\perp}}{\beta_{k}^{\perp}+2 \beta_{k}^{\|}} .
$$

Figure 3 compares $\beta_{k} / \beta_{1}$ evaluated using $\beta_{k}^{\|} / \beta_{1}$ and $\beta_{k}^{\perp} / \beta_{1}$ from the ratio of the appropriate collision rates Eq. (12) and Eq. (17) (crosses) to the direct calculation of $\beta_{k} / \beta_{1}$ (diamonds) via Eq. (5) and (10). The agreement is very satisfactory. 

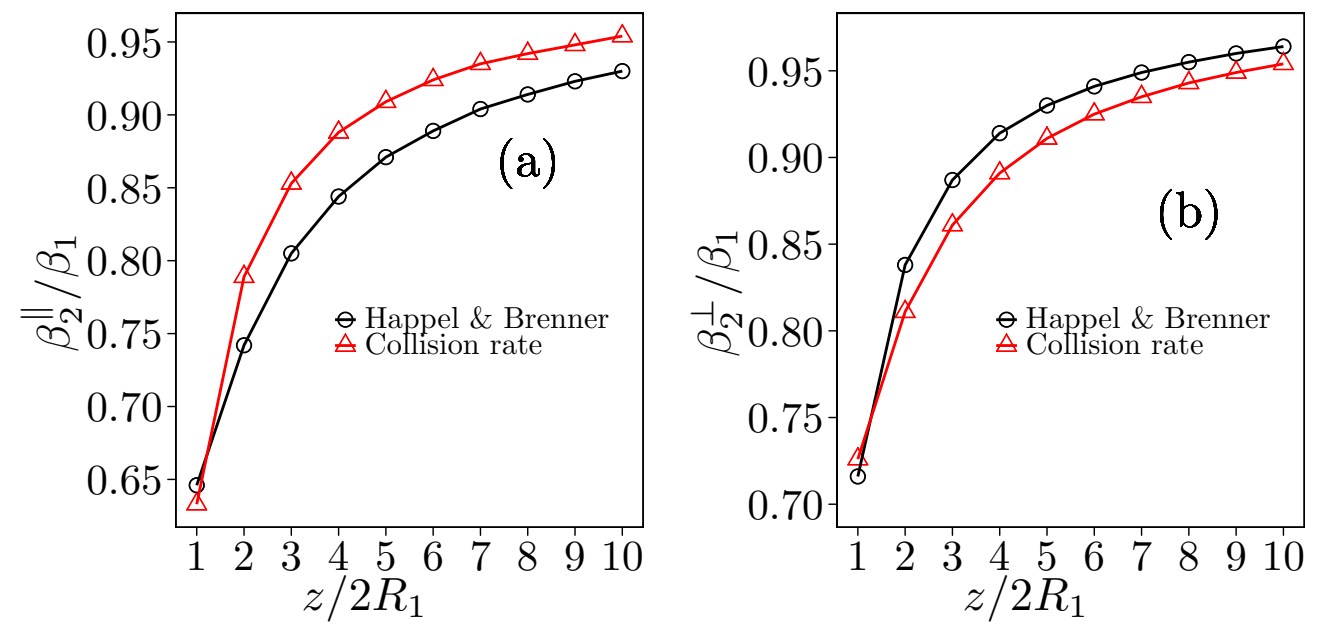

Figure 2: Calculated parallel (a) and perpendicular (b) friction coefficients of two equalsized, non-rotating spheres as a function of their center-of-mass distance $z$. Comparison with the results of Happer \& Brenner [7].

The mobility radius $R_{k}$ of straight chains calculated by Eq. (7) is shown in Fig. 4(a). As expected, the mobility radii of chains composed of shielded monomers are considerably smaller than the mobility radii of ideal chains [for which $R_{k} \sim k$, Eq. (7)]. In addition, their radius of gyration $R_{g}$ is plotted. It is easy to show that the radius of gyration of straight $k$-chains is

$$
R_{g}^{2}=\frac{1}{3} R_{1}^{2}\left(k^{2}-1\right)+R_{1}^{2} .
$$

The last term $R_{1}^{2}$ is an additional term to the usual definition of the radius of gyration to ensure that the radius of gyration of a monomer is non-zero. The choice shown in Eq. (18) corresponds to the geometric radius of a sphere [6]; alternatively, the radius of gyration of a sphere $\left(R_{1} \sqrt{3 / 5}\right)$ has been used [13].

The ratio of the mobility diameter to the radius of gyration is shown in Fig. 4(b). Note that for straight chains the two diameters differ significantly, as also noted in Refs. [6, 20]. They argued that the usual assumption $R_{k} \sim$ $R_{g}$ may be justified for dense fractal-like aggregates with fractal dimension greater than 2, but the approximate equality fails for smaller dimensions, in agreement with our results shown in Fig 4(b) for straight chains. For large straight chains in the limit $k \rightarrow \infty$ Eqs. (15) (with the constants determined from the fit of the collision-rate friction coefficients) and Eqs. (17, 18) show 


\begin{tabular}{|c|c|c|c|c|}
\hline \multirow[t]{2}{*}{$\begin{array}{l}\text { Number of } \\
\text { monomers }\end{array}$} & \multirow{2}{*}{$\begin{array}{l}\text { Average dynamic } \\
\text { shape factor }\left(\chi_{k}\right) \\
\text { Collision } \\
\text { rate }\end{array}$} & \multirow{2}{*}{$\begin{array}{l}\text { Parallel dynamic } \\
\text { shape factor }\left(\chi_{k}^{\|}\right) \\
\text {Collision } \\
\text { rate }\end{array}$} & \multicolumn{2}{|c|}{$\begin{array}{l}\text { Perpendicular dynamic } \\
\text { shape factor }\left(\chi_{k}^{\perp}\right)\end{array}$} \\
\hline & & & $\begin{array}{l}\text { Collision } \\
\text { rate }\end{array}$ & $\begin{array}{l}\text { Filippov } \\
\text { Ref. } 6\end{array}$ \\
\hline 2 & 1.102 & 1.005 & 1.152 & 1.15 \\
\hline 3 & 1.194 & 1.041 & 1.274 & 1.276 \\
\hline 4 & 1.278 & 1.083 & 1.379 & 1.386 \\
\hline 5 & 1.354 & 1.126 & 1.472 & 1.485 \\
\hline 8 & 1.556 & 1.250 & 1.713 & \\
\hline 16 & 1.975 & 1.533 & 2.203 & \\
\hline 24 & 2.312 & 1.769 & 2.592 & \\
\hline 32 & 2.601 & 1.975 & 2.924 & \\
\hline 40 & 2.862 & 2.162 & 3.223 & \\
\hline 48 & 3.100 & 2.334 & 3.495 & \\
\hline 56 & 3.322 & 2.495 & 3.748 & \\
\hline 64 & 3.531 & 2.647 & 3.987 & \\
\hline
\end{tabular}

Table 2: Average dynamic shape factor $\left(\chi_{k}\right)$ of straight chains, and their dynamic shape factor for motion parallel $\left(\chi_{k}^{\|}\right)$and perpendicular $\left(\chi_{k}^{\perp}\right)$ to their symmetry axis. The last column reports results of Ref. [6].

that the ratio of the mobility radius to the radius of gyration tends to

$$
\frac{R_{k}}{R_{g}}=1.05 \frac{\sqrt{3}}{\ln (2 k)} \quad \text { as } \quad k \rightarrow \infty \quad,
$$

in good agreement with the slender-body theory prediction [6, 24]

$$
\frac{R_{k}}{R_{g}}=\frac{\sqrt{3}}{\ln (2 k)}+O\left(\frac{1}{\ln ^{2}(2 k)}\right) .
$$

Equations (16) reproduce the slender-body theory result.

The connection between collision rates and the average monomer shielding factor may be refined to obtain the shielding factor of a monomer within an aggregate. The intra-chain (isotropic, per unit monomer mass) friction coefficient $\beta_{k}^{(i)}$ of monomer $i$ in a $k$-aggregate is defined by

$$
\frac{K_{k}^{(i)}}{K_{1}}=\frac{\beta_{k}^{(i)}}{\beta_{1}} \equiv \eta_{k}^{(i)}
$$




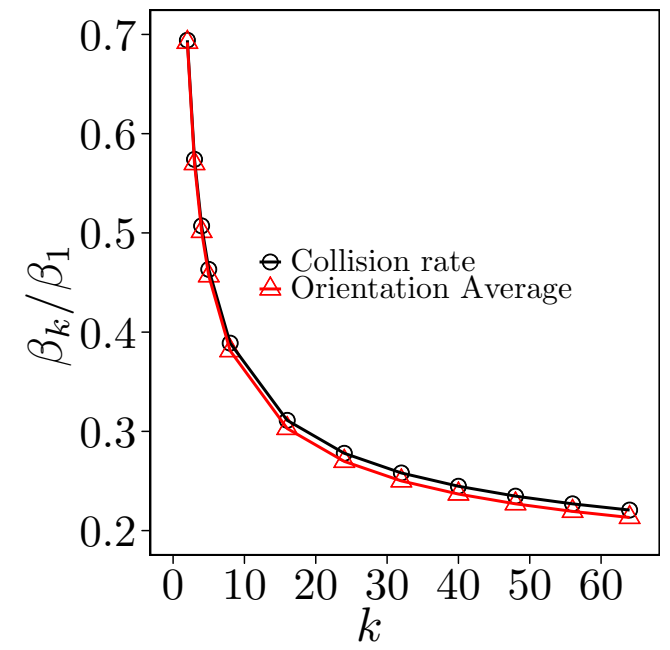

Figure 3: Isotropic straight-chain shielding factors $\eta_{k}=\beta_{k} / \beta_{1}$ calculated via the total collision rates from the diffusion simulations (diamonds) and from anisotropic collision rates and Eq. (17) (triangles) $(k=2-64)$.

where $K_{k}^{(i)}$ is the steady-state molecular collision rate of the $i$ th monomer in the $k$-chain. It is easy to show that

$$
\eta_{k}=\frac{1}{k} \sum_{i=1}^{k} \eta_{k}^{(i)} \quad \Longrightarrow \quad f_{k}=\sum_{i=1}^{k} f_{k}^{(i)}=\sum_{i=1}^{k} \eta_{k}^{(i)} f_{1}
$$

where $f_{k}$ is the total aggregate friction coefficient, $f_{k}^{(i)}$ the total friction coefficient of the $i$ th monomer, and $f_{1}=m_{1} \beta_{1}$ the monomer friction coefficient. Equation (21), as Eq. (5), can be applied to aggregates of arbitrary shape. Table 3 presents position-dependent monomer shielding factors in $k=5,8$ straight chains.

\begin{tabular}{ccc|cccc}
\hline \hline$\eta_{5}^{(1)}$ & $\eta_{5}^{(2)}$ & $\eta_{5}^{(3)}$ & $\eta_{8}^{(1)}$ & $\eta_{8}^{(2)}$ & $\eta_{8}^{(3)}$ & $\eta_{8}^{(4)}$ \\
\hline 0.597 & 0.379 & 0.364 & 0.565 & 0.350 & 0.325 & 0.317 \\
\hline \hline
\end{tabular}

Table 3: Monomer shielding factors in two straight chains, $k=5,8$. The superscript denotes the relative position of the monomer in the chain, the subscript the total number of monomers. 

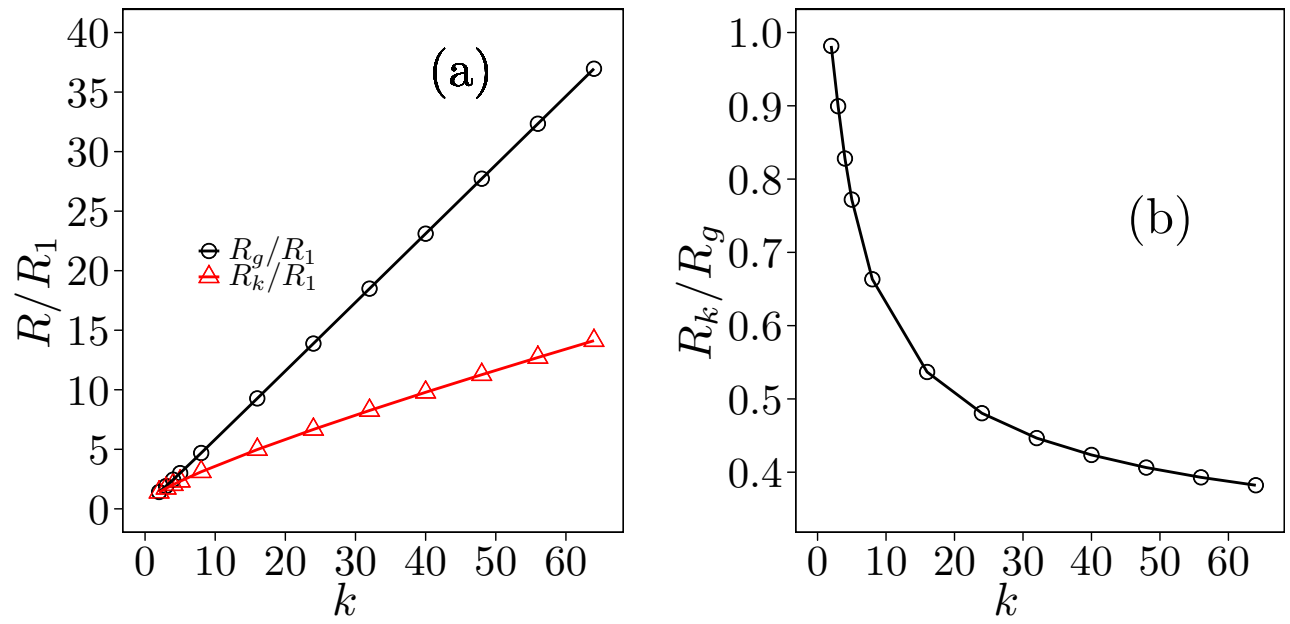

Figure 4: Left: Dimensionless straight-chain mobility diameter $R_{k} / R_{1}$ (triangles) and radius of gyration $R_{g} / R_{1}$ (circles) plotted against number of monomers $(k=2-64)$. Right: Ratio of the mobility radius to the radius of gyration of straight chains as a function monomer number $(k=2-64)$.

\section{Langevin dynamics of straight-chain aggregates}

Langevin simulations have been used extensively to investigate aggregate collisional dynamics, see, for example, Refs. [13, 25], and in particular the diffusive motion of aggregates. The Brownian motion of an aggregate may be described by modelling the Brownian motion of a set of interacting monomers held together by strong monomer-monomer interaction forces. A coupled set of Langevin equations, each one for a monomer within the aggregate, is solved to determine the diffusive motion of the whole aggregate. The stochastic properties of random force, which describes the effect of molecular collisions with the aggregate, are usually determined by assuming that the Fluctuation Dissipation Theorem (FDT) hold for each monomer. Accordingly, the random force acting on each monomer is determined from its friction coefficient. The monomer friction coefficient is usually assumed to be independent of aggregate morphology and equal to the average monomer friction coefficient $\beta_{k}$. In particular, in the free draining approximation (i.e., for ideal clusters) the monomer friction coefficient is taken to be the friction coefficient of an isolated monomer [13. In the continuum regime, these assumptions yield the aggregate diffusion coefficient $D_{k}$ as given in Eq. (6). 
The previously described determination of the intra-chain shielding factor allows the introduction of a monomer-dependent random force. In the following we use these intra-chain shielding factors to calculate the diffusion coefficient of straight chains from the Langevin dynamics of each monomer in the chain. The Langevin equations of motion of the $i$ th monomer in a $k$-chain is

$$
m_{1} \ddot{\mathbf{r}}_{i}=\mathbf{F}_{i}-\beta_{k}^{(i)} m_{1} \dot{\mathbf{r}}_{i}+\mathbf{W}_{i}(t)
$$

where $\mathbf{r}_{i}$ is the monomer position, $\mathbf{F}_{i}$ the external force acting on it, and $\mathbf{W}_{i}$ the random force that models the effect of molecule-monomer collisions. The stochastic properties of the noise are usually taken to be delta-correlated in time and space $\left(j, j^{\prime}=x, y, z\right.$; monomers are identified by $\left.i, i^{\prime}\right)$

$$
A_{\perp} / A_{\|}\left\langle W_{i}^{j}(t)\right\rangle=0,\left\langle W_{i}^{j}(t) W_{i^{\prime}}^{j^{\prime}}\left(t^{\prime}\right)\right\rangle=\Gamma_{i} \delta_{i i^{\prime}} \delta_{j j^{\prime}} \delta\left(t-t^{\prime}\right)
$$

The monomer-dependent noise strength $\Gamma_{i}$ is determined from the FDT to be

$$
\Gamma_{i}=2 \beta_{k}^{(i)} m_{1} k_{B} T,
$$

explicitly depending on the monomer shielding factor. The corresponding noise strength in Langevin simulations where the average monomer friction coefficient $\beta_{k}$ is used becomes $\Gamma=2 \beta_{k} m_{1} k_{B} T$, i.e., it is independent of the monomer position in the chain.

The force in Eq. (23) models monomer-monomer interactions. It will be taken to be conservative

$$
\mathbf{F}_{i}=-\nabla_{\mathbf{r}_{i}} U_{i}
$$

where $U_{i}$ is the total intermonomer potential the $i$ th monomer feels. The monomer potential will be chosen to ensure the stiffness of the straight chain. We split potential into a radial part $U_{i}^{\text {rad }}$ and an angular part $U_{i}^{\text {ang }}$. The radial potential fixes the distance between any two first-neighbor monomers along the chain, and the angle-dependent potential $U_{i}^{\text {ang }}$ prevents the chain from bending. The radial part is taken to be pairwise additive

$$
U_{i}^{\mathrm{rad}}=\sum_{j \neq i}^{k} u_{i j}\left(r_{i j}\right)
$$

where $r_{i j}=\left|\mathbf{r}_{i}-\mathbf{r}_{j}\right|$. In our simulations the radial, two-body potential $u_{i j}\left(r_{i j}\right)$ was chosen to be the pair-wise additive model potential described in 
Ref. [13. The angular potential $U_{i}^{\text {ang }}$ depends on the angle $\phi_{i, i-1, i+1}$ formed by the three consecutive monomers $(i-1, i, i+1)$ as follows

$$
U_{i}^{\text {ang }}=\Omega\left[1-\cos \left(\phi_{i, i-1, i+1}-\pi\right)\right], i=2, \ldots, k-1,
$$

where $\Omega$ is a constant that determines the bending stiffness of the chain. Figure 5 illustrates the definition of $\phi_{i, i-1, i+1}$ within a $k=5$ chain. The monomer-monomer potential described by Eqs. (27) and (28) introduces a penalty function for any radial or angular deformation of the linear chain to ensure that the chain remain straight and rigid during its Brownian motion.

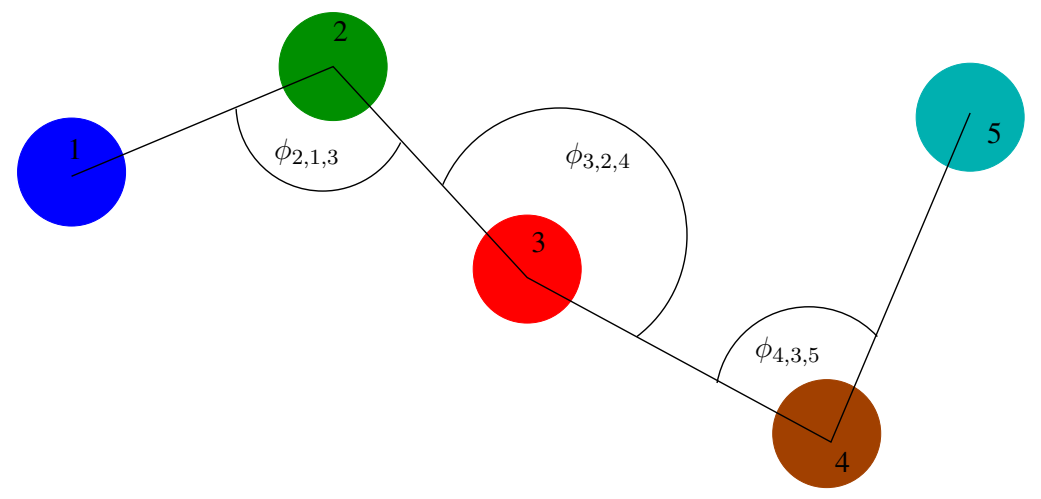

Figure 5: Definition of the angles used in the angular monomer-monomer interaction potential $U_{i}^{\text {ang }}$ for a linear chain of $k=5$ monomers.

We performed the numerical simulations of the mobility of straight chains with the molecular dynamics package ESPResSo [26], modified to allow for a monomer-dependent random force. The depth of the radial inter-monomer potential was chosen deep enough to ensure that monomers remain bound. Similarly, the strength of the angular potential $\Omega$ was chosen to ensure that the linear chain did not bend during the simulation $\left(\Omega=500 k_{B} T\right)$.

The chain diffusion coefficient $D_{k}^{L D}$ was estimated from the time dependence of the chain centre-of-mass mean-square displacement

$$
\lim _{t \rightarrow \infty}\left\langle\delta r_{C M}^{2}(t)\right\rangle=6 D_{k}^{L D_{t}},
$$

where brackets denote an ensemble average. We followed the motion of the aggregate centre-of-mass along 3200 trajectories up to $\beta_{1} t=100$ for chains of $k=5,8$. The chain was placed in the middle of a box of size $L$ with 
$L /\left(2 R_{1}\right)=10000$. The box size was large enough so that no overall displacement of the aggregate larger than $L / 2$ was observed. Details of similar simulations are described in Ref. [13].

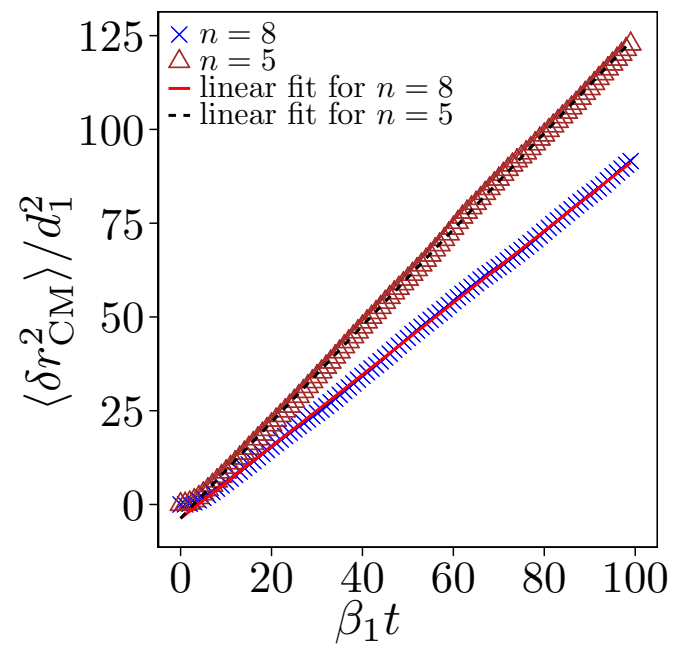

Figure 6: Normalized mean-square displacements of the center-of-mass of straight chains consisting of $k=5,8$ monomers ( $d_{1}$ is the monomer diameter). The linear fits were performed for $\beta_{1} t \geq 3$.

The dimensionless chain center-of-mass displacements (scaled by the monomer diameter $d_{1}$ ) are shown in Fig. 6 as a function of dimensionless time for the two straight chains. The numerical fit of the linear dependence of $\left\langle\delta r_{\mathrm{CM}}^{2}\right\rangle$ on time was performed for $\beta_{1} t \geq 3$. We find $D_{5}^{L D} / D_{1}=0.428$ and $D_{8}^{L D} / D_{1}=$ 0.319 , were $D_{1}$ is the monomer diffusion coefficient. These values are within $1 \%$ of the diffusion coefficient $D_{5} / D_{1}=0.432$ and $D_{8} / D_{1}=0.321$ obtained from Eq. (6) and the shielding factors reported in Table 1, i.e., via the average monomer shielding factor calculated from the total collision rates. Since the diffusion coefficient is inversely proportional to the friction coefficient these results confirm numerically that the aggregate friction coefficient is the sum of the fiction coefficient of the shielded monomers, Eq. (22).

Therefore, the Langevin simulations provide additional support to the proposed methodology whereby the diffusion coefficient of straight chains may be accurately calculated via the ratio of molecular collision rates. Furthermore, they suggest that calculations of $k$-aggregate diffusion may be performed by two different, but equivalent, approaches. An average monomer 
friction coefficient $\beta_{k}=\eta_{k} \beta_{1}$ may be used on each monomer in the aggregate, as in usual Langevin simulations via, e.g., ESPResSo. The FDT is assumed to hold for an average monomer and the noise strength is the same for all monomers $\Gamma=2 \beta_{k} m_{1} k_{B} T$. Alternatively, individual monomer friction coefficients $\beta_{k}^{(i)}(i=1, \ldots k)$ are introduced, and the complete set of monomer-dependent friction coefficients $\left\{\beta_{k}^{(i)}\right\}$ (or, equivalently, $\left\{\eta_{k}^{(i)}\right\}$ ) has to be specified. The FDT is, again, assumed to hold for each monomer, but the noise strength becomes monomer-dependent $\Gamma_{i}=2 \beta_{k}^{(i)} m_{1} k_{B} T$, Eq. 23). Both descriptions of aggregate Brownian motion are consistent because they yield the same diffusion coefficient $D_{k}$.

\section{Conclusions}

We developed a methodology to calculate approximately, albeit accurately, the friction and diffusion coefficients of generic fractal-like aggregates via the ratio of molecule-aggregate collision rates. The collision rates are obtained from numerical simulations of the molecular diffusion equation with an absorbing boundary condition on the aggregate surface. The methodology was validated for straight chains via comparison of friction coefficients, isotropic and anisotropic, with analytical calculations and extrapolations of experimental measurements. Since the numerical solution of the diffusion equation (a scalar Laplace equation) is often more feasible than sophisticated treatments of the Stokes equations relatively accurate insights on aggregate mobility and monomer shielding factors may be gained from collision-rate simulations.

In addition to the average shielding factor of a monomer in an aggregate, the collision rate on each monomer was used to define a monomerdependent intra-chain friction coefficient. Langevin-dynamics simulations of the diffusive motion of straight chains were performed to argue that within our approximation the Fluctuation Dissipation Theorem could be equally well applied on the whole aggregate by considering an average monomer or on each monomer, as long as the appropriate shielding factors, and consequently the friction coefficients, are used. The diffusion coefficients of straight chains composed of $k=5,8$ monomers calculated by Langevin simulations were found to be in good agreement with the diffusion coefficients calculated via the ratio of the molecular collision rates. The Langevin simulations depended explicitly on the inter-monomer interaction potential, thereby providing a useful technique to investigate the effect of inter-particle forces on 
agglomeration dynamics and on the shape of the resulting aggregates.

\section{Acknowledgements}

We thank Anastasios Melas for useful discussion and additional collisionrate simulations.

\section{References}

[1] S.K. Friedlander, Smoke, Dust and Haze, Oxford University Press, New York, 2000.

[2] P.L. Garcia-Ybarra, J.L. Castillo, D.E. Rosner, J. Aerosol Sci. 37 (2006) 413.

[3] M. Vanni, Chem. Eng. Sci. 55 (2000) 685.

[4] P. Vainshtein, M. Shapiro, C. Gutfinger, J. Aerosol Sci. 35 (2004) 383.

[5] S. Veerapaneni, M.R. Wiesner, J. Colloid Inter. Sci. 177 (1996) 45.

[6] A.V. Filippov, J. Colloid Interface Sci. 229 (2000) 184.

[7] J. Happel, H. Brenner, Low Reynolds number hydrodynamics, 2nd edn., Kluwer Academic Press, Dordrecht, Holland, 1991.

[8] B. Dahneke, Aerosol Sci. Technol. 1 (1982) 179.

[9] R.K. Chakrabarty, H. Moosmüller, W. Patrick Arnott, M.A. Garro, G. Tian, J.G. Slowik, E.S. Cross, J.-H. Han, P. Davidovits, T.B. Onasch, D.R. Worsnop, Phys. Rev. Lett. 102 (2009) 235504; 104 (2010) 119602; M. Sander, R.I.A. Patterson, A. Raj, M. Kraft, ibid. 104 (2010) 119601;

[10] S. Di Stasio, A.G. Konstandopoulos, M. Kostoglou, J. Colloid Inter. Sci. 247 (2002) 33.

[11] M. Kostoglou, A.G. Konstandopoulos, J. Aerosol Sci. 32 (2001) 1399.

[12] M. Kostoglou, A.G. Konstandopoulos, S.K. Friedlander, J. Aerosol Sci. 37 (2006) 1102.

[13] L. Isella, Y. Drossinos, Phys. Rev E 82 (2010) 011404. 
[14] K. Siegmann, H.C. Siegmann, Society of Automotive Engineers Technical Paper (2000) 2000-01-1995.

[15] A. Keller, K. Siegmann, A.C. Siegmann, A. Filippov, J. Vacuum Sci. Technol. A 19 (2001) 1.

[16] W.C. Hinds, Aerosol Technology, 2nd edn., Wiley \& Sons, New York, 1999.

[17] W.A. Heitbrink, D.E. Evans, B.K. Ku, A.D. Maynard, T.J. Slavin, T.M. Peters, J. Occup. Environ. Hyg. 6 (2009) 19.

[18] F. Reif, Fundamentals of Statistical and Thermal Physics, McGraw-Hill, New York, 1965.

[19] R.C. Sonntag, W.B. Russel, J. Colloid Inter. Sci. 115 (1987) 378.

[20] A.V. Filippov, M. Zurita, D.E. Rosner, J. Colloid Interface Sci. 229 (2000) 261.

[21] P. Résibois, M. de Leener, Classical Kinetic Theory of Fluids, John Wiley \& Sons, New York, 1977.

[22] T.V. Elzhov and K.M. Mullen, Minpack.lm: $R$ interface for least squares optimization library, http://cran.r-project.org/web/ packages/minpack.lm/index.html (2008).

[23] Comsol Multiphysics, Chemical Engineering Module, version 3.5, http: //www. comsol.com/products/chem/ (2008).

[24] G.K. Batchelor, J. Fluid Mechanics 44 (1970) 419.

[25] R.D. Mountain, G.W. Mulholland, H. Baum, J. Colloid Interface Sci. 114 (1986) 67.

[26] H.J. Limbach, A. Arnold, B.A. Mann, C. Holm, Computer Physics Communications 174 (2006) 704. 